\title{
Experimental Study of Multi Optical Parameter Imaging Technology under the Fog Condition
}

\author{
Weiwei Feng, Ligang Chen \\ College of Aeronautical Engineering, Binzhou University, Binzhou, China \\ Email: fwwclg@126.com, clgwlx@126.com
}

How to cite this paper: Feng, W.W. and Chen, L.G. (2017) Experimental Study of Multi Optical Parameter Imaging Technology under the Fog Condition. Optics and Photonics Journal, 7, 86-91. https://doi.org/10.4236/opj.2017.78B012

Received: May 30, 2017

Accepted: August 7, 2017

Published: August 10, 2017

\begin{abstract}
A multi optical parametric imaging system is introduced and established in order to improve the contrast of object in the fog. A few targets are observed in the fog weather based on the system level radiation model of multi optical parametric imaging and the calibrated model parameters. The results show that the building's windows can be distinguished clear in the linear polarization, circular polarization and angle of polarization images because of the strong reflected polarization light of the glass; The vehicles in intersection can hardly be seen in the intensity image, and it is fuzzy in degree of linear polarization and angle of polarization image because of the doped polarization information of trees near in fog; The circular polarization image raises the contrast of the vehicles by $20 \%$ because the circle polarization of the trees is less in the fog.
\end{abstract}

\section{Keywords}

Multi Optical Parameter, Radiation Model, Polarization Imaging

\section{Introduction}

Recently, there are so Frequent Haze weather of many cities in China which affects not only people's health but also the visibility and influence People's Daily life at the same time, especially in the fields of the road traffic, sea targets monitoring and aviation flight safety [1] [2] [3]. Many scholars have carried out the research on the target identification of smog weather. Using the basic principle of atmospheric scattering model and combining with the sunny weather of contrast image, K Nayar drawn from several images of different concentration of fog scene structure, to achieve image enhancement purpose in 1999 [4]. In 2000, David B et al. carried out the optical polarization imaging experiment in smoke, and the results showed that the polarization imaging improved the contrast ratio 
of the image in the scattering medium [5]. Schechner, et al. Studied the parallel and vertical polarization direction of scene images in haze weather successively and analyze the target image, the result showed that image contrast [6] are increased obviously. Wang Yong and Tianquan Liang carried out the study of the linear polarization image recognition method by using $0^{\circ}, 60^{\circ}, 120^{\circ}$ which are three different polarization directions of polarization radiation pattern, the experimental results showed the method can more effectively to distinguish the distant goal, to improve the target image contrast and obtain the target distance information [7] [8].

Polarization remote sensing application in the field of astronomy is the earliest use of polarization camera to get pictures of the earth's surface polarization, the observation of different vegetation, and mineral surface polarization information work [9]. Light polarization information can be expressed by Stokes vector (I, Q, U, V) T, in which I is the intensity of polarized light, $\mathrm{Q}, \mathrm{U}$ represent $\mathrm{x}$ linear polarized light and $\mathrm{y}$ linear polarized light, $\mathrm{V}$ represents the circularly polarized light [10]. In current polarization remote sensing detection, the circular polarization information is often ignored. For example, the EOSP instrument in the United States and POLDER in France only use polarizer to measure radiation information in different polarization directions, so only the first three Stokes parameters [11] [12] can be calculated. In order to get the circular polarization information, the phase delay device is needed [13], such as cirrus detector (CCS) system which is developed by the Chinese academy of sciences Shanghai technology cirrus detector (CCS) with rotating quarter wave plate, the measuring system is a full polarization measurement without imaging which is aimed cirrus clouds as the main target and detection of other types of clouds and atmospheric aerosols [14]. Compared with radiation intensity or linear polarization degree of image detection technology which are based on single parameter information, multiple optical parametric measurement imaging system this paper developed can simultaneously obtain the target's multiple optical parametric image information such as intensity, polarization radiation, the partial radiation, linear polarization, circular polarization degree and polarization Angle, etc. target recognition ability in the fog haze image can be improved by the test of the multidimensional data analysis.

\section{Multi-Optic Parametric Measurement Imaging System and Its Principle}

Multiple imaging optical parametric measurement system is built based on full polarization camera, the system can acquire the incident beam's Stokes vector (S0 and S1, S2, S3), and the target's multi-dimensional image information such as radiation intensity and polarization, the polarization radiation, linear polarization degree, degree of circular polarization and polarization Angle and so on can also be obtained through the analysis of the image data processing. As shown in Figure 1, the system mainly includes optical imaging lens, achromatic 


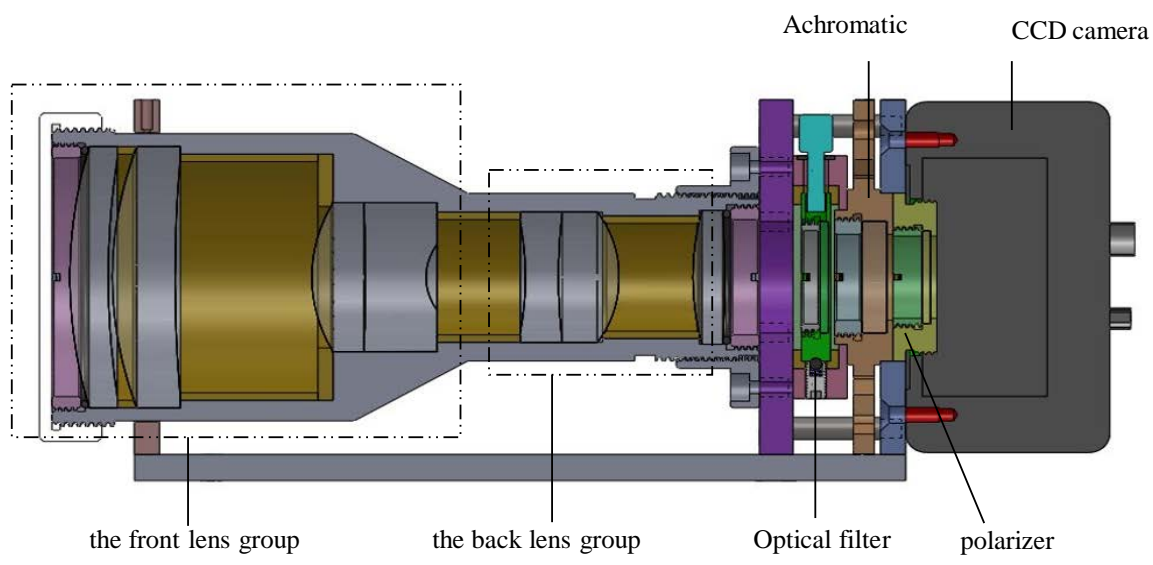

Figure 1. The multi optical parameter imaging system.

quarter wave plate, polarizer, CCD surface array detector and image data processing software. The optical imaging lens is composed of the front group and the positive lens of the negative lens. The whole system is designed to minimize the imaging beam direction multiple optical parametric measurement error caused by the problem.

The direction of the optical axis of the multiple imaging optical parametric measurement system is Z-axis, and the transmission axis of plolarizer instrument is the $\mathrm{X}$-axis, as shown in Figure 2.

According to the imaging propagation of polarization beam in optical polarization system, the radiation response of multi-optical parametric imaging system is obtained [15] [16]:

$$
X=\vec{P} \cdot \vec{S}+C
$$

where $\vec{S}=\left(S_{0}, S_{1}, S_{2}, S_{3}\right)$ is the Stokes vector for the incident beam.

$$
\text { And } \vec{P}=A\left(\begin{array}{c}
q+r+\cos (2 \alpha)(q-r) \\
\cos (2 \alpha)(q-r)+\cos ^{2}(2 \alpha)(q+r)+2 \sin ^{2}(2 \alpha) \sqrt{q \cdot r} \cos (\delta) \\
\sin (2 \alpha)(q-r)+\sin (4 \alpha)\left(\frac{q+r}{2}-\sqrt{q \cdot r} \cos (\delta)\right) \\
-2 \sin (2 \alpha) \sqrt{q \cdot r} \sin (\delta)
\end{array}\right) \text { is }
$$

the measuring vector of the imaging system. Where $A$ is absolute radiation calibration factor, $\alpha$ is the quick axial wave plate in absolute Angle in the coordinate system of the system. $q$ and $r$ are the transmission ratio of the two direction which are fast and slow axis respectively. Their ratio reflects the wavelength dichroism. $\delta$ is the actual phase delay of the achromatic lambda/4 wave plate. $C$ is dark current correction factor.

In this experiment measurement, the fast axis $\alpha$ in the coordinate system of the absolute Angle can varies continuously by rotating the achromatic $\lambda / 4$ wave plate. And the radiation intensity output response can be obtained, and the linear measurement equation can be established as show in the Equation (2):

$$
X_{\alpha}{ }^{\prime}=\vec{P}_{\alpha} \cdot \vec{S}
$$




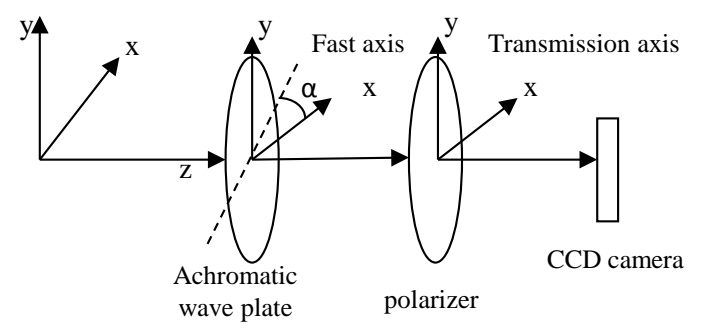

Figure 2. The images of relative intensity transmission coefficient.

where $\vec{S}$ is the normalized Stokes vector of the incoming beam. $\alpha$ takes six polarization angles $(0, \pi / 6, \pi / 3 \pi / 2,2 \pi / 3$ and $5 \pi / 6), X_{\alpha}{ }^{\prime}=X_{\alpha}-\overline{\mathrm{C}}$ is The output of the radiation measured when the average amount of dark current is deducted. The Stokes vector (I, Q, U, V) T of the incoming beam can be measured by Matrix operation Equation (2) and then the multi-optic parameters of the target are obtained such as intensity I, polarization radiation Ip, non-polarization radiation Inon, linear polarization degree LP, circular polarization degree $\mathrm{CP}$ and polarization Angle $\theta$.

\section{Multi-Optical Parametric Measurement Imaging Experiments}

Using the multi-optic parametric measurement imaging system, we conducted a series of experiments on the smog of the sky, buildings and traffic intersections, As shown in Figure 3. The imaging work band is $750 \mathrm{~nm}$.

Normally atmospheric media has strong polarization scattering properties, and its polarization scattering intensity is related to scattering Angle. In our haze weather scenario experiment, the distance the sky 1) is a large Angle backscatter, so the non-polarization radiation of the atmospheric scattering light radiation intensity is strong and polarization radiation is relatively weak. Building glass 2) of the total reflection energy is very weak, but the polarization of reflected light is relatively strong, we can all distinguish the window structure more clearly in the figures of linear polarization, circular polarization, polarization Angle. The non-polarization radiation of distant buildings 3 ) is stronger, and it mixed atmospheric background light as a whole, so it is not easy to find. But the distant glass of building with little non-polarization radiation can be used to identify the target. In the scene of our experiment, the trees 4 ) has a certain amount of degree of linear polarization relative to the surrounding background, but its circularly polarized radiation information in haze weather has no particularly advantages of distinguish. For the cars at traffic intersection 5) the location, it is difficult to identify these distant cars in the intensity figure. But they can be seen in the figures of the polarized radiation, linear polarization diagram and polarization Angle, but the polarization information of nearby trees, makes the vehicle target is fuzzy. In the circularly polarized radiation figure, circular polarization information the nearby trees is less, so the vehicle target contrast can be increased by $20 \%$, and the vehicle at the junction can be distinguish clearly. 


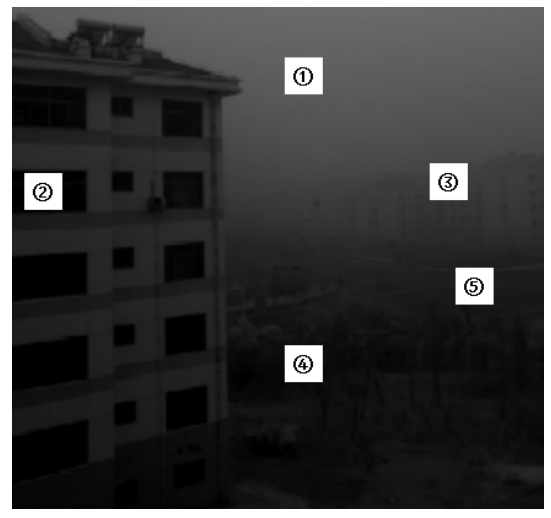

(a)

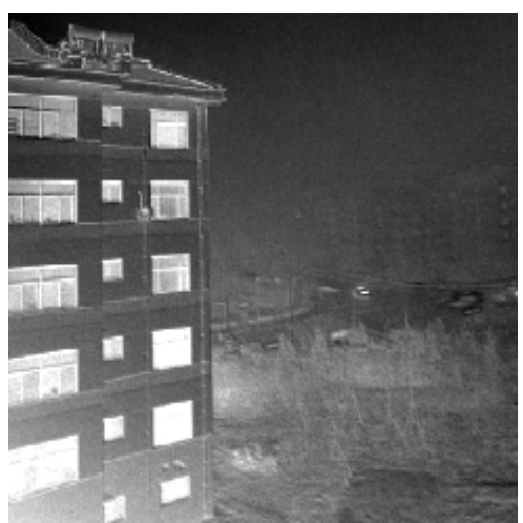

(d)

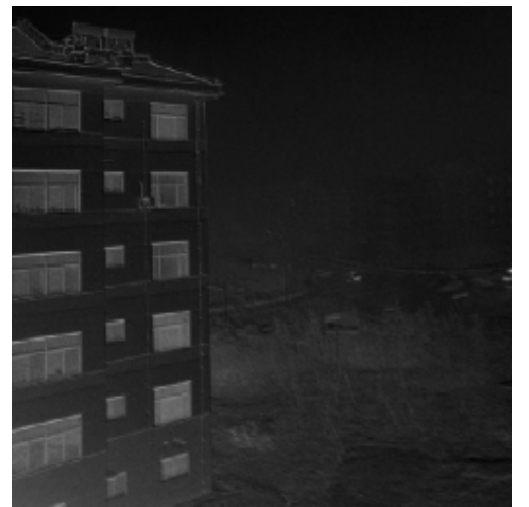

(b)

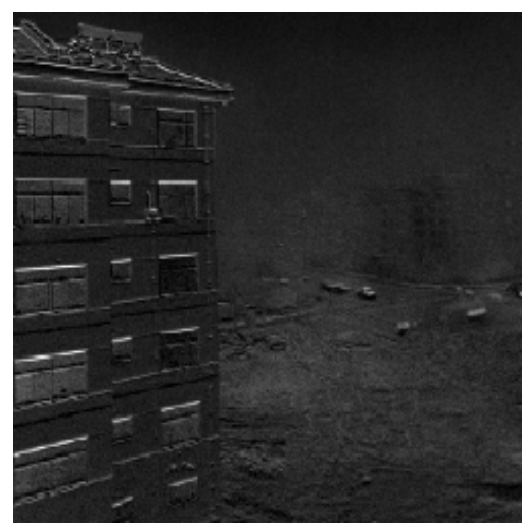

(e)

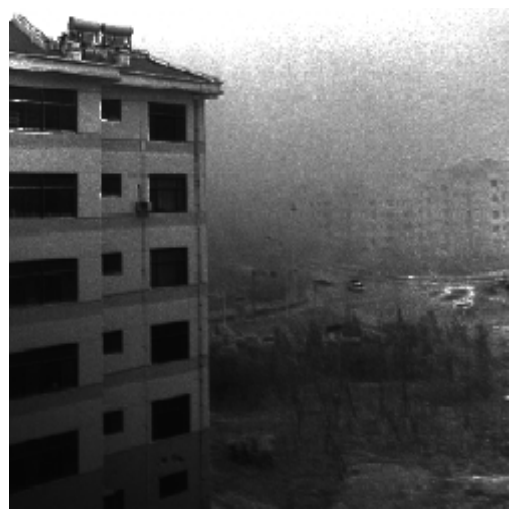

(c)

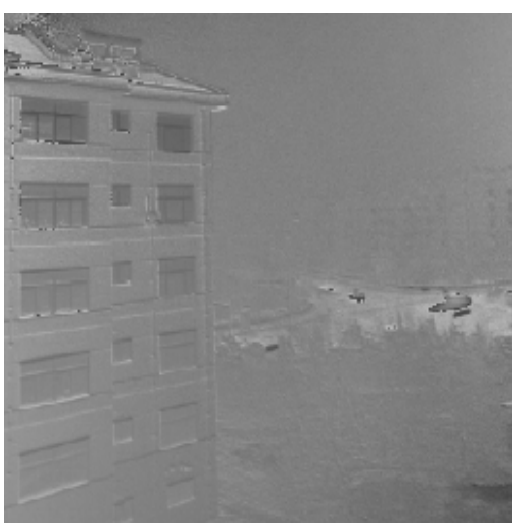

(f)

Figure 3. The multi optical parametric imaging effect under the fog. (a) The intensity figure; (b) Polarized radiation figure; (c) non-polarization radiation figure; (d) Linear polarization figure; (e) Circular polarization figure; (f) The polarization Angle of figure.

\section{Conclusion}

There are multiple scientific research teams which carry out the haze image target recognition research, basing on the conventional radiation and spectral information, and the light of linear polarization information (mainly the linear polarization degree or two orthogonal polarization direction) is also gradually introduced. In this paper, a new target recognition method based on multiple optical parametric information is put forward, a multiple optical parametric imaging system is established, and it is used to measure multiple optical parametric information of target in fog haze weather conditions. The optical parametric information includes strength, polarized radiation, the partial radiation, linear polarization degree, circular polarization degree, polarization Angle, etc. However, this approach is limited by many factors such as the error from the design, process and the parameter calibration of Multi-optic parametric measurement imaging system. And it is also limited by External cause, such as fog haze type, concentration, and observation date/time, observation angle caused which will bring he uncertainty on the target recognition with the uncertainty, these factors will be discussed in subsequent papers. 


\section{Acknowledgements}

This work is partly financially supported by two National Natural Science Foundation of China (Grant No. 41401384 and Grant No.41201368). This work is also financially supported by Science and technology development project of Shandong Province (Grant No. J14LJ02) and Research Foundation Program of Binzhou University (grant No. 2013Y09).

\section{References}

[1] Zhao, G.X., Du, L., Wei, L.P., et al. (2011) Comprehensive Analysis on a Durative Regional Haze and Fog. Arid Zone Research, 28, 871-878.

[2] Song, Y., Tang, X.Y., Fang, C., et al. (2003) Relationship between the Visibility Degradation and Particle Pollution in Beijing. Acta Scientiae Circumstantiae, 23, 468471.

[3] Sheng, L.F., Liang, W.F., Qu, W.J., et al. (2001) Relationship of Aerosol Size Distribution and Visibility in a Sea Fog. Periodical of Ocean University of China, 41, 1-8.

[4] Nayar, S.K. and Narasimhan, S.G. (1999) Vision in Bad Weather. The Proceedings of the Seventh IEEE International Conference on Computer Vision, 2, 820. https://doi.org/10.1109/ICCV.1999.790306

[5] David, B.C.J. and Larry, P. (2000) Polarization Imaging through Scattering Media. Proc. SPIE, 4133, 124-133. https://doi.org/10.1117/12.406619

[6] Schechner, Y.Y., Srinivasa, G.N. and Shree, K.N. (2003) Polarization-Based Vision through Haze. Applied Optics, 42, 511-525. https://doi.org/10.1364/AO.42.000511

[7] Wang, Y., Xue, M.G. and Hang, Q.C. (2009) Polarization Dehazing Algorithm Based on Atmosphere Background Suppression. Computer Engineering, 35, 271275.

[8] Liang, T.Q., Zhao, Q., Sun, X.B., et al. (2014) Research on Image Restoration by Polarized Remote; Sensing through Haze. Geomatics and Information Science of Wuhan University, 39, 244-247.

[9] Shi, Z.H. (2002) Polarization Imaging Measurement Technology and Its Application. Infrared, 4, 1-5.

[10] Chen, L.G. (2015) Polarimetric Calibration of the Polarization CCD Camera with Large Viewing Field. Opto-Electronic Engineering, 42, 15-20.

[11] Deschamps, P.Y., Herman, M., Podaire, A., et al. (1992) The POLDER Instrument: Mission Objectives. SPIE, 746, 72-91.

[12] Travis, L.D. (1992) Remote Sensing of Aerosols with the Earth Observing Scanning Polarimeter. SPIE, 1747, 154-164.

[13] Feng, W.W., Ma, G.L., Jia, T.J., et al. (2011) Measurement of Complete Stokes Parameters of 2-D Plolarized Light. Laser Technology, 35, 715-717.

[14] Shao, W.D., Wang, P.G., Zheng, Q.B., et al. (2003) Polarimetric Calibration of Airborne Remote Sensing Polarimeter. J. Infrared Millim. Waves, 22, 137-140.

[15] Chen, L.G. and Feng, W.W. (2015) Study on System-Level Calibration of Achromatic $\lambda / 4$ Wave-Plate. Journal of Applied Optics, 36, 905-908. https://doi.org/10.5768/JAO201536.0603001

[16] Azzam, R.M.A. and Bashara, N.M. (1986) Ellipsometry and Polarized Light. Science Press, Beijing, 37-40. 
Submit or recommend next manuscript to SCIRP and we will provide best service for you:

Accepting pre-submission inquiries through Email, Facebook, LinkedIn, Twitter, etc. A wide selection of journals (inclusive of 9 subjects, more than 200 journals)

Providing 24-hour high-quality service

User-friendly online submission system

Fair and swift peer-review system

Efficient typesetting and proofreading procedure

Display of the result of downloads and visits, as well as the number of cited articles Maximum dissemination of your research work

Submit your manuscript at: http://papersubmission.scirp.org/

Or contactopj@scirp.org 